Full length article

\title{
Co-immobilization of Palm and DNase I for the development of an effective anti-infective coating for catheter surfaces
}

\author{
Diana Alves $^{\mathrm{a}, *}$, Andreia Magalhães ${ }^{\mathrm{a}}$, Daria Grzywacz ${ }^{\mathrm{b}}$, Damian Neubauer ${ }^{\mathrm{c}}$, Wojciech Kamysz ${ }^{\mathrm{b}, \mathrm{c}}$, \\ Maria Olívia Pereira ${ }^{a}$ \\ ${ }^{a}$ CEB - Centre of Biological Engineering, LIBRO - Laboratório de Investigação em Biofilmes Rosário Oliveira, University of Minho, Campus de Gualtar, 4710-057 Braga, Portugal \\ ${ }^{\mathrm{b}}$ Peptideweb.com, 80-298 Gdansk, Poland \\ ${ }^{\mathrm{c}}$ Faculty of Pharmacy, Medical University of Gdansk, 80-416 Gdansk, Poland
}

\section{A R T I C L E I N F O}

Article history:

Received 12 April 2016

Received in revised form 23 June 2016

Accepted 7 August 2016

Available online 8 August 2016

\section{Keywords:}

Catheter-associated infections

Bi-functional coating

Dopamine chemistry

Bacterial resistance

Cytotoxicity

\begin{abstract}
A B S T R A C T
Biomaterial-associated infections, in particular, catheter-associated infections (CAI) are a major problem in clinical practice due to their ability to resist antimicrobial treatment and the host immune system. This study aimed to co-immobilize the antimicrobial lipopeptide Palm and the enzyme DNase I to introduce both antimicrobial and anti-adhesive functionalities to polydimethylsiloxane (PDMS) material, using dopamine chemistry. Surface characterization confirmed the immobilization of both compounds and no leaching of Palm from the surfaces for up to 5 days. Co-immobilization of both agents resulted in a bifunctional coating with excellent surface antimicrobial and anti-biofilm properties against both Staphylococcus aureus and Pseudomonas aeruginosa. The modified surfaces demonstrated superior biocompatibility. To better discriminate co-adhesion of both species on modified surfaces, PNA FISH (Fluorescence in situ hybridization using peptide nucleic acid probes) was employed, and results showed that $P$. aeruginosa was the dominant organism, with $S$. aureus adhering afterwards on $P$. aeruginosa agglomerates. Furthermore, Palm immobilization exhibited no propensity to develop bacterial resistance, as opposite to the immobilization of an antibiotic. The overall results highlighted that co-immobilization of Palm and DNase I holds great potential to be applied in the development of catheters.
\end{abstract}

\begin{abstract}
Statement of Significance
Catheter-associated infections (CAI) are the most common hospital-acquired infections worldwide. Several coating strategies have been proposed to fight these infections but most of them present some important limitations, including the emergence of resistant bacteria and toxicity concerns. The present work describes a two-step polydopamine-based surface modification strategy to successfully coimmobilize an antimicrobial peptide (Palm) and an enzyme targeting an important component of biofilm matrix (DNase I). This immobilization approach imparted polydimethylsiloxane surfaces with both antiadhesive and antimicrobial properties against the adhesion of relevant bacteria as single and dualspecies, with excellent stability and biocompatible and anti-biofilm properties, holding, therefore, great potential in the development of catheters able to prevent CAI.
\end{abstract}

(c) 2016 Acta Materialia Inc. Published by Elsevier Ltd. All rights reserved.

\section{Introduction}

Catheter-associated infections are the infections most frequently acquired in a clinical context as a result of the general use of both urinary and intravascular catheterization [1]. These infections are often associated to biofilm formation [2,3], a microconsortia of surface adhering cells enclosed in a self-produced

\footnotetext{
* Corresponding author.

E-mail address: dianalves@ceb.uminho.pt (D. Alves).
}

polymeric matrix [4], essentially comprised of water, polysaccharides, proteins and extracellular DNA. This extracellular matrix is greatly responsible for biofilms success as a survival strategy of microorganisms, since it affords mechanical support, architectural stability, mediates cell-cell and cell-surface interactions and acts as a protective barrier [5]. Treatment procedures for patients suffering from CAI usually involve intravenous and oral antibiotic therapy, in which high doses can be administered for several weeks or months [6]. Emerging microbial resistance to widely prescribed antibiotics compromises the success of this approach and, very 
often, the treatment fails, and the only solution for the infected catheter is its surgical removal, at the expenses of patient suffering and considerable costs [7].

The development of novel approaches to fight CAI is, thus, in great demand. As bacterial adhesion plays an important role in biofilm formation, several surface modifications intended to render the surfaces with the ability to resist bacterial colonisation have been proposed, including catheter impregnation with compounds such as silver or antibiotics [8,9]. There are, however, crucial limitations associated to both strategies, such as the emergence of multi-drug resistant bacteria and toxicity concerns [10]. Antimicrobial peptides (AMP) and enzymes that target biofilm matrix components have been accepted as promising alternative candidates to impart biomaterial surfaces with antibacterial features [11-13]. Several approaches have been applied for the immobilization of active compounds onto the surfaces of biomaterials including covalent chemical conjugation and physical adsorption [14]. There are, however, some limitations associated to them as typical chemical conjugation methods involve several steps that complicate the conjugation process and may reduce the immobilization efficiency $[15,16]$. An alternative approach, which has been attracting considerable interest in the last few years, is the polydopamine (pDA) coating, which is based on a mussel adhesive protein that is rich in 3,4-dihydroxyphenylalanine (L-DOPA) and L-lysine enabling these organisms to tightly attach themselves to wet surfaces. Messersmith and co-workers have showed that selfpolymerization of dopamine, a molecule that combines both functionalities of catechol and amine, under oxidative and alkaline conditions, results in the deposition of a thin adherent polymer film (pDA) on different types of material surfaces [17]. This pDA layer can subsequently act as a versatile platform for immobilization of active compounds. For example, pDA coating has been used to immobilize CWR11, a synthetic AMP, onto PDMS as well as onto a commercial catheter, providing the surfaces with potent antimicrobial and anti-biofilm properties, excellent stability and biocompatibility properties [18]. Polydopamine coating has also proved to be a versatile platform for the immobilization of lysostaphin, a peptidoglycan hydrolase enzyme able to hydrolyse the covalent bonds in bacterial cell walls, causing their lysis. Enzyme's immobilization did not compromise its activity yielding surfaces able to effectively kill S. aureus and prevent biofilm formation [19].

The aim of the current study was to co-immobilize the AMP Palm and the enzyme DNase I onto PDMS, using dopamine chemistry. It was intended to obtain a bi-functional coating combining both anti-adhesive and antimicrobial properties, with the ability to prevent biofilm formation, without cytotoxicity or propensity to develop bacterial resistance.

\section{Materials and methods}

\subsection{Bacterial strains and growth conditions}

Staphylococcus aureus GB 2/1 isolated from explanted voice prostheses at the University Medical Centre of Groningen (The Netherlands) was used throughout this study as a model strain. A reference strain of Pseudomonas aeruginosa (ATCC 39324) was also used to reinforce some experiments. The strains were first streaked on a tryptic soy agar (TSA, Merck) plate from a frozen stock solution and grown for $24 \mathrm{~h}$ at $37^{\circ} \mathrm{C}$. For each experiment, a few colonies were collected from the TSA plates and grown overnight in batches of tryptic soy broth (TSB, Merck) at $37^{\circ} \mathrm{C}$ under agitation $(120 \mathrm{rpm})$. Subsequently, cells were harvested by centrifugation ( $9000 \mathrm{~g}, 5 \mathrm{~min}$, at room temperature) and washed three times in phosphate buffer saline (PBS, $10 \mathrm{mM}$ potassium phosphate, $150 \mathrm{mM} \mathrm{NaCl}, \mathrm{pH}$ 7.4). The cellular suspension was adjusted by optical density (OD) at $640 \mathrm{~nm}$ and calibrated by means of colony forming units (CFU).

\subsection{AMP and enzyme}

The enzyme DNase I was purchased by AppliChem (Germany) and the AMP Palm-KGK-NH $\mathrm{N}_{2}$ (Palm) was synthesized manually by solid-phase synthesis method on polystyrene AM-RAM resin, using Fmoc/tButyl strategy [20]. Coupling was performed with HOBt/DIPCDI method, the Fmoc protecting group were removed with $20 \%$ piperidine. Crude peptides were cleaved from resin using a mixture of trifluoroacetic acid (TFA), triisopropylsilane (TIS) and water as scavengers. The final products were purified by reversephase high performance liquid chromatography (RP-HPLC) in a mixture of acetonitrile - water with $0.1 \%$ TFA as an eluent. Molecular weights of peptides were determined by matrix - assisted laser desorption ionization-time of flight mass spectrometry (MALDI-TOF).

\subsection{Antimicrobial activity of Palm}

The minimal inhibitory (MIC) and bactericidal (MBC) concentrations of Palm were determined by the microdilution method according to Clinical and Laboratory Standards Institute (formerly NCCLS) [21]. Briefly, the wells of a sterile 96-well round-bottom microtiter plates (polystyrene, Orange, USA) were filled with $100 \mu \mathrm{L}$ of Mueller Hinton Broth (MHB, Merck) with increasing concentrations of peptide to which were added $100 \mu \mathrm{L}$ of each bacterial suspension (adjusted to a final concentration of $5.0 \times 10^{5} \mathrm{CFU} / \mathrm{mL}$ ). The plates were afterwards incubated at $37^{\circ} \mathrm{C}$ for $24 \mathrm{~h}$ in an orbital shaker at $120 \mathrm{rpm}$. In this assay, two controls were used, one without bacteria as a negative control and one without peptide as a positive control. The MIC of the planktonic fraction was obtained by measuring the $\mathrm{OD}$ at $640 \mathrm{~nm}\left(\mathrm{OD}_{640 \mathrm{~nm}}\right)$ in an automated microtiter plate reader (Sunrise, Tecan), where clear wells $\left(\mathrm{OD}_{640 \mathrm{~nm}}=\right.$ negative control $)$ were evidence of bacterial growth inhibition. MBC determination was performed by adding a droplet of $10 \mu \mathrm{L}$ from each well with no visible growth on a TSA plate. The lowest concentration that yielded no colony growth after $24 \mathrm{~h}$ at $37^{\circ} \mathrm{C}$ was identified as the MBC. Three independent assays with six replicates for each condition were performed.

\subsection{PDMS preparation}

PDMS was prepared by mixing and curing the two-component kit Sylgard 184 (Dow Corning, USA) according to the manufacturer's instructions. Briefly, base and curing agents provided in the kit were combined in 10:1 $(\mathrm{w} / \mathrm{w})$, cast in a petri dish and kept at room temperature for $48 \mathrm{~h}$. The cured PDMS was then cut into circle pieces of $0.9 \mathrm{~cm}$ diameter at a thickness of approximately $0.3 \mathrm{~cm}$. Prior utilization, coupons were sonicated in a commercial detergent (Sonasol, Henkel Ibérica, Portugal) for about $5 \mathrm{~min}$, rinsed with distilled water for a few minutes, sonicated in methanol for about $20 \mathrm{~min}$, then rinsed with distilled water and air-dried overnight.

\subsection{Polydopamine coating and further functionalization}

Coatings were prepared according to a two-step method previously developed for immobilizing enzyme into magnetic nanoparticles [22]. Briefly, the first step involved the deposition of a pDA coating onto PDMS coupons which was performed by immersing them in $7 \mathrm{~mL}$ of a freshly prepared solution of dopamine (Sigma, St. Louis, Missouri; $2 \mathrm{mg} / \mathrm{mL}$ dopamine- $\mathrm{HCl}$ in $10 \mathrm{mM}$ bicine buffer, $\mathrm{pH} 8.5$ ) for $18 \mathrm{~h}$ at room temperature and 
under agitation (70 rpm). Coupons were then rinsed with ultrapure water. For further functionalization, pDA-coated coupons were immersed in a DNase I solution $(1 \mathrm{mg} / \mathrm{ml}$ in $150 \mathrm{mM} \mathrm{NaCl}$, $10 \mathrm{mM}$ potassium phosphate buffer supplemented with $10 \mathrm{mM}$ $\mathrm{MgCl}_{2}$, pH 6.8, pDA-DNase I) or in Palm solution $(1 \mathrm{mg} / \mathrm{mL}$ in PBS, $\mathrm{pH}$ 7.4, pDA-Palm) and were incubated for $6 \mathrm{~h}$, at room temperature under agitation (70 rpm). Co-immobilization was performed by immersing pDA-coated coupons in a mixture solution composed of DNase I and Palm (pDA-MIX). The proportion of each antimicrobial in the mixture solution was optimized and details can be found in the Supplementary Material (Fig. S1). After peptide and/or enzyme immobilization, the coupons were rinsed with ultrapure water.

\subsection{Surface characterization}

The surface morphology of the materials was analysed by scanning electron microscopy (SEM). Prior to observation, samples were sputter coated with gold and observed with an S-360 scanning electron microscope (Leo, Cambridge, MA, USA). SEM imaging was performed with the following parameters: $15 \mathrm{kV}$ accelerating voltage, $22 \mathrm{~mm}$ stage distance, $500 \times$ and $5000 \times$ magnification. The surface morphology and roughness were also evaluated using atomic force microscopy (AFM). AFM measurements were performed at room temperature using a Multimode with a Nanoscope III from Digital Instruments (USA) operating in tapping mode. Scan rates were set at $1 \mathrm{~Hz}$ and the scanning area per sample was fixed at $5 \mu \mathrm{m} \times 5 \mu \mathrm{m}$. Surface morphology and roughness analysis were conducted using NanoScope Analysis 1.10 software. Surface wettability was investigated by measuring static water contact angle of the PDMS after each deposition step by a sessile drop method using an automated contact angle measurement apparatus (OCA 15 Plus, Dataphysics, Germany) that allows image acquisition and data analysis. Contact angles were measured using $3 \mu \mathrm{L}$ drops of water. Three independent assays with three replicates for each condition were performed.

\subsection{Palm immobilization efficiency and stability}

To determine the efficiency of Palm immobilization, the supernatants containing loaded and unattached peptide were collected in the beginning and in the end of coating process, and quantified using a fluorescamine (Sigma) assay [23]. Fluorescamine assay was performed by mixing fluorescamine solution ( $3 \mathrm{mg} / \mathrm{mL}$ in acetone) and the sample at 1:3 ratio in a 96-black-well plate (Greiner). Samples were then incubated for $15 \mathrm{~min}$ at room temperature, in the dark and the fluorescence intensity was measured using a microplate reader (Synergy HT, Biotek). Immobilization efficiency was represented as the percentage obtained from the ration between the amount of immobilized peptide and the amount of loaded peptide. Three independent assays with three replicates for each condition were performed. To investigate coatings stability, namely compounds releasing from the surfaces, the amount of released peptide from the modified surfaces were measured under a physiologically relevant condition (in PBS at $37^{\circ} \mathrm{C}$ ). For that, $500 \mu \mathrm{L}$ of a fresh PBS solution was added to each well of a 48-well microtiter plate (Orange Scientific, USA) in which PDMS coupons functionalized with Palm were placed immediately after peptide immobilization. The coupons were then incubated at $37^{\circ} \mathrm{C}$ for 5 days. Every day, supernatant samples $(500 \mu \mathrm{L})$ were withdrawn to determine the amount of peptide released. The remaining peptide on the surface was quantified by subtracting the released peptide from the total amount of peptide immobilized at the first day. These experiments were performed twice with three replicates for each condition tested.

\subsection{Bacterial viability on modified PDMS surfaces}

Antibacterial performance of the generated surfaces against bacterial adhesion was evaluated by preparing a bacterial suspension with $1 \times 10^{8} \mathrm{CFU} / \mathrm{mL}$ in PBS from an overnight culture at $37{ }^{\circ} \mathrm{C}$. For dual-species adhesion, a combination of $50 \%$ of suspended inoculum of each species was used. PDMS coupons were placed into the wells of a 48-well tissue culture plate (Orange Scientific, USA) and covered with $300 \mu \mathrm{L}$ of bacterial suspension. The samples were kept at $37^{\circ} \mathrm{C}$ and $120 \mathrm{rpm}$ for $4 \mathrm{~h}$, as it has been reported to be enough to achieve a complete initial adhesion to a surface $[24,25]$. Samples were then washed with saline solution $(0.9 \% \mathrm{NaCl})$, stained with a live/dead stain (BacLight Bacterial Viability Kit, Invitrogen) and observed in a fluorescent inverted microscope (Leica, DMI 3000B). In this assay, the red-fluorescent nuclei acid staining agent propidium iodide, which only penetrates damaged cell membranes, was used to label dead bacterial cells. Contrariwise, the SYTO 9 green-fluorescent nucleic acid staining agent, which is able to penetrate cells whether with intact or compromised membranes, was used to label viable cells. At least three images per coupon (a single image for each fluorescence channel) were collected and at least 3 coupons were inspected per assay. Image (Version $1.49 \mathrm{~m}$, Wayne Rasband, National Institutes of Health, USA) software was used to subtract the image background and the threshold function was used to render each greyscale image into a binary translation with distinct areas identifying adhered bacteria. The threshold value supplied by ImageJ was used as default. However, if necessary, this value was manually adjusted so that all the cells could be included within the thresholded range. The area measurement function was then used to determine the area of the pixels above the threshold and, in this way, to quantify the area covered by bacteria discriminating, at the same time, the fraction of live and dead bacteria, depending on the channel being analysed. Values were normalized to PDMS control. At least two independent assays with three replicates for each condition were performed.

\subsection{Localization and distribution of bacterial populations by PNA FISH}

In order to assess bacterial spatial organization and the species distribution on the coated surfaces, PNA FISH (peptide nucleic acid fluorescence in situ hybridization) method was employed. Before starting the hybridization, co-adhesion of $P$. aeruginosa and S. aureus was allowed to occur for $4 \mathrm{~h}$ as previously described. Coupons were then washed with saline solution and air-dried for $15 \mathrm{~min}$. Bacteria were fixed with methanol (100\%) for $20 \mathrm{~min}$. This fixation step is important to prevent bacterial detachment during hybridization procedure [26]. Fixed bacteria were stored at $4{ }^{\circ} \mathrm{C}$ for no longer than $48 \mathrm{~h}$ before the multiplex PNA FISH procedure. A specific 16S rRNA PNA probe (Paer565) previously developed [27] was used for $P$. aeruginosa detection and $S$. aureus was identified by counterstaining the samples with 4, 6-diamidino-2phenylindole (DAPI, Sigma) at the end of the hybridization procedure. After bacteria fixation with methanol, $20 \mu \mathrm{L}$ of $4 \%(\mathrm{w} / \mathrm{v})$ paraformaldehyde followed by $50 \%$ ethanol $(\mathrm{v} / \mathrm{v})$ were applied to cover the entire surface and incubated for $10 \mathrm{~min}$ each and allowed to air dry. This step enables the fixation of the cells and increase the permeabilization of the cell membrane to the subsequent hybridization allowing the labelled oligonucleotide probes to diffuse to their intracellular rRNA target molecules [28]. Afterwards, $20 \mu \mathrm{L}$ of hybridization solution containing the probe at $200 \mathrm{nM}$ was applied on coupons which were covered with coverslips and incubated in the dark for $1 \mathrm{~h}$ at $65^{\circ} \mathrm{C}$. After hybridization, coupons were inserted in a 24 -well plate (Orange Scientific, USA) containing a pre-warmed (at $65^{\circ} \mathrm{C}$ ) washing solution composed of $5 \mathrm{mM}$ Tris Base, $15 \mathrm{mM} \mathrm{NaCl}$ and $0.1 \%$ (v/v) Triton X-100 (Sigma). The plate 
was incubated for $30 \mathrm{~min}$ at $65^{\circ} \mathrm{C}$ in the dark. Finally, coupons were allowed to air dry in the dark before counterstaining with DAPI $(40 \mu \mathrm{g} / \mathrm{mL})$ for $5 \mathrm{~min}$ at room temperature in the dark and cells were visualized under an epifluorescence microscope. For visualization, a fluorescence microscope (Olympus BX51, Perafita, Portugal) equipped with the filters sensitive to DAPI (BP 365-370, FT 400, LP 421) and to the signalling molecule of the PNA probe (BP 530-550, FT 570, LP 591) were used. Three independent assays with three replicates were performed.

\subsection{Evaluation of biofilm formation by XTT reduction assay}

In order to investigate the potential of the modified coatings to impair biofilm formation, the metabolic activity of biofilm cells was evaluated using the 2,3-bis (2-methoxy-4-nitro-5-sulfophe nyl)-2H-tetrazo-lium-5-carboxanilide sodium salt (XTT, Sigma) colorimetric method as described by Stevens and Olsen [29], with some modifications. Briefly, a bacterial suspension with $1 \times 10^{7} \mathrm{CFU} / \mathrm{mL}$ was prepared in TSB and $300 \mu \mathrm{L}$ of this suspension were added to a 48-well microtiter plate in which PDMS surfaces were placed. The plate was incubated for $24 \mathrm{~h}$ at $37^{\circ} \mathrm{C}$, at $120 \mathrm{rpm}$. The coupons were subsequently washed with saline solution to remove free-floating bacteria and $400 \mu \mathrm{L}$ of a combined solution of XTT and phenazine methosulfate (PMS, Sigma) were added to each well in order to obtain a final concentration of $150 \mu \mathrm{g} / \mathrm{mL}$ of XTT and $10 \mu \mathrm{g} / \mathrm{ml}$ of PMS. The plates were afterwards incubated at $37^{\circ} \mathrm{C}$ for $3 \mathrm{~h}$, at $120 \mathrm{rpm}$, in the dark. The OD of each well was measured at $490 \mathrm{~nm}$ using a microtiter plate reader (Tecan, Model Sunrise-basic Tecan, Austria). Two independent assays with three replicates were performed.

\subsection{Susceptibility of adhered cells to modified surfaces}

In order to evaluate the susceptibility pattern of bacterial cells adhered to PDMS surfaces, cells in contact with modified and unmodified surfaces were recovered and allowed to adhere to new bare and modified samples during a period of 10 days. Briefly, a bacterial suspension with $1 \times 10^{7} \mathrm{CFU} / \mathrm{mL}$ was prepared in TSB and $300 \mu \mathrm{L}$ of this suspension were added to a 48 -well microtiter plate (Orange Scientific, USA) in which PDMS coupons were placed. The plate was incubated for $24 \mathrm{~h}$ at $37^{\circ} \mathrm{C}$ and $120 \mathrm{rpm}$. The coupons were subsequently washed 3 times with saline solution to remove free-floating bacteria and transferred to an eppendorf tube with $1 \mathrm{~mL}$ of TSB. The tubes were then sonicated for $6 \mathrm{~min}$, gently subjected to vortex for $30 \mathrm{~s}$ and $300 \mu \mathrm{L}$ of this suspension were added to a 48-well microtiter plate in which new PDMS coupons were placed. The procedure was repeated for 10 successive days. The MIC and MBC for each condition tested were determined and compared. As a control, the antibiotic vancomycin (Sigma) was immobilized onto PDMS using dopamine chemistry, at the same concentration as Palm. Experiments were performed in triplicate.

\subsection{Cytotoxicity assay}

Cytotoxicity tests were performed according to the ISO 109935:2006, using fibroblast cells 3T3 (CCL 163) obtained from ATCC, commonly used for biomaterial surface biocompatibility studies [30,31]. Cells were first cultured in Dulbecco's modified Eagle's medium (DMEM) supplemented with $10 \%$ of fetal bovine serum and $1 \%$ penicillin/streptomycin at $37{ }^{\circ} \mathrm{C}, 5 \% \mathrm{CO}_{2}$. After achieving the confluence, cells were detached using trypsin and $500 \mu \mathrm{L}$ of a cell suspension with $1 \times 10^{5}$ cells/mL were added to each well of a 48-well microtiter plate in which the PDMS surfaces were previously inserted. The plates were incubated at $37^{\circ} \mathrm{C}, 5 \% \mathrm{CO}_{2}$ for $48 \mathrm{~h}$. After that period of time, cytotoxicity was evaluated by the MTS (3-(4,5-dimethylthiazol-2-yl)-5-(3-carboxymethoxyphenyl)-2(4-sulfophenyl)-2H-tetrazolium), inner salt reduction assay as a measure of cellular metabolic viability. All the medium was removed and a solution containing $100 \mu \mathrm{L}$ of MTS (Promega CellTiter $96^{\circledR}$ AQueous NonRadioactive Cell Proliferation Assay) per each $1 \mathrm{~mL}$ of DMEM without phenol red was added to each well. After $1 \mathrm{~h}$ of incubation in the dark, at $37^{\circ} \mathrm{C}, 5 \% \mathrm{CO}_{2}$, the absorbance of the resulting solution was red at $\mathrm{OD}_{490 \mathrm{~nm}}$. Results were expressed as percentage of viable cells using the metabolic activity of cells grown on pDA-coated PDMS as controls.

\subsection{Statistical analysis}

Results were presented as mean \pm standard deviation (SD). Statistical analysis was performed by Kolmogorov-Smirnov normality test using Graph Pad Prism 5.0 for Mackintosh. Afterwards, parametric tests (one way Anova followed by Tukey's test) or nonparametric (Kruskal-Wallis test) were implemented, depending on whether the samples were from normally distributed populations or not, respectively.

\section{Results}

\subsection{MIC and MBC determination on planktonic cultures}

The concentrations of Palm able to inhibit planktonic bacterial growth (MIC) and those required to kill planktonic bacteria (MBC) of $S$. aureus were $32 \mu \mathrm{g} / \mathrm{mL}$ and $64 \mu \mathrm{g} / \mathrm{mL}$, respectively. MIC and MBC of Palm against P. aeruginosa were both $64 \mu \mathrm{g} / \mathrm{mL}$.

\subsection{Co-immobilization of DNase I and Palm onto PDMS}

Surface modification involved the deposition of a uniform coating of pDA from a dopamine- $\mathrm{HCl}$ solution at a slightly alkaline $\mathrm{pH}$. The pDA coating was then used as a platform for Palm and/or DNase I immobilization due to the presence of residual quinones which present convenient sites for covalent grafting of nucleophilic groups such as amine and/or thiol functional groups found in both peptide and enzyme via Michael Addition and/or Shiff reactions, as well as via physical adsorption. For co-immobilization, a mixture solution composed of enzyme and AMP at different proportions were investigated and a proportion of $1: 3$, respectively, yielded the best combination of both anti-adhesive and antimicrobial properties (Fig. S1).

\subsection{Surface characterization}

Surface morphology of modified PDMS surfaces was characterized using SEM analysis. The unmodified PDMS surfaces presented a smoother morphology compared with the modified ones (Fig. 1A). Self-polymerized polydopamine particles could be observed on modified PDMS coupons confirming the pDA coating. Further functionalization with Palm and/or DNase I yielded surfaces with different morphologies, depending on the compound immobilized. Surfaces functionalized with DNase I present a rougher surface morphology as compared to the ones with Palm, which is most probably due to the fact that DNase I is inherently larger in size. Co-immobilization of both compounds (pDA-MIX) yields surfaces with an intermediate morphology. For further surface morphology characterization, samples were also analysed by AFM (Fig. 1B). AFM results confirmed that bare PDMS possessed a smoother morphology as compared to modified surfaces. From the AFM images, it was possible to measure the average roughness of surfaces (Fig. 2A) and results indicated that the presence of a pDA layer increased the surface roughness of PDMS, which is in 
A)
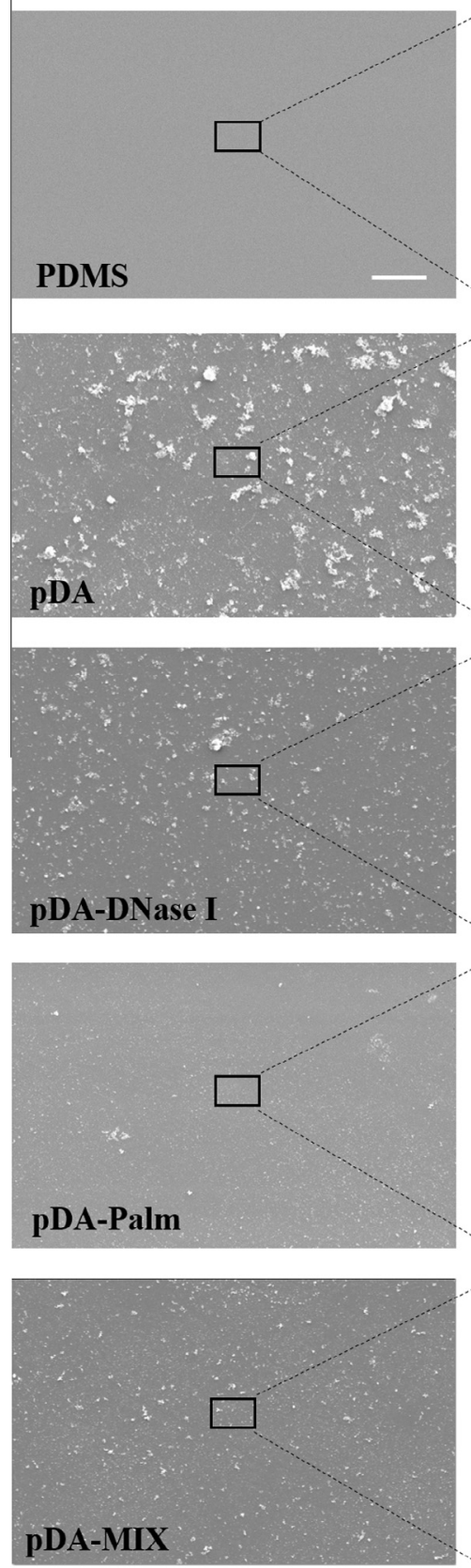
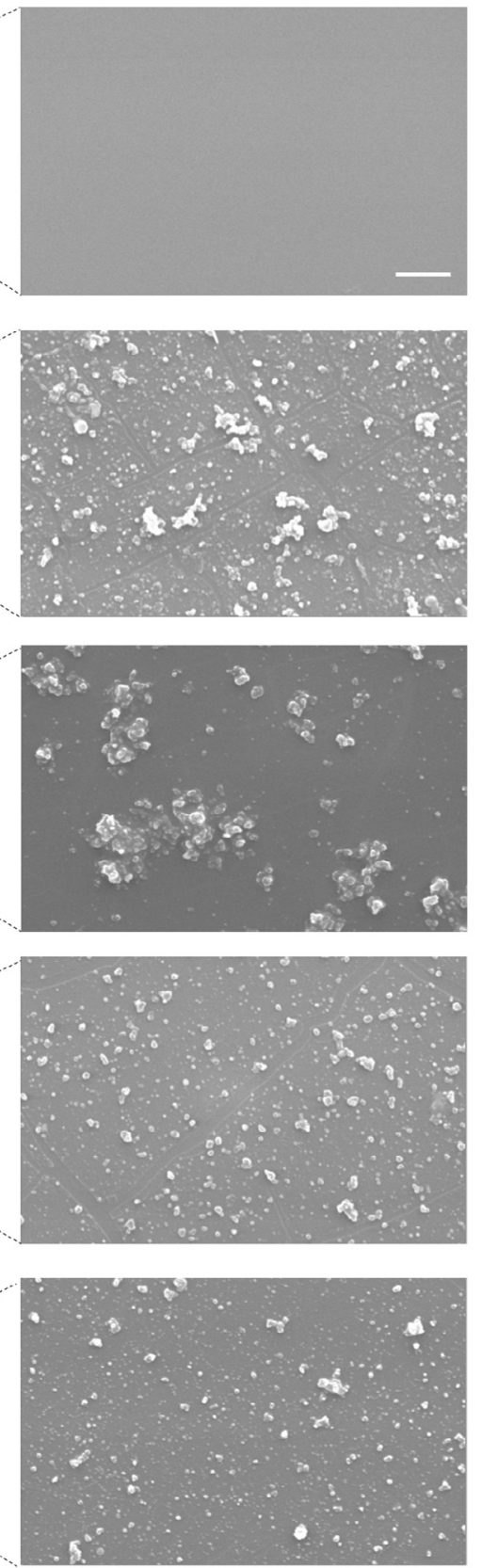

B)
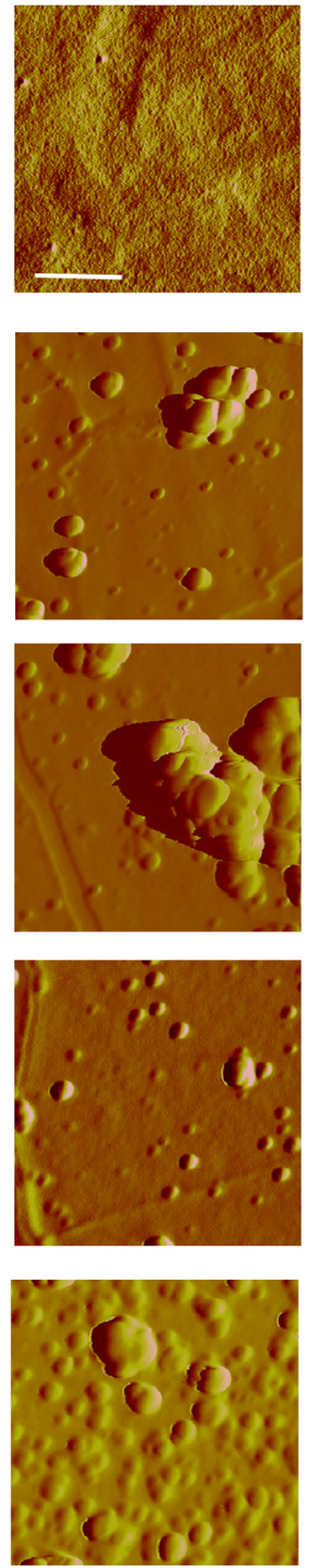

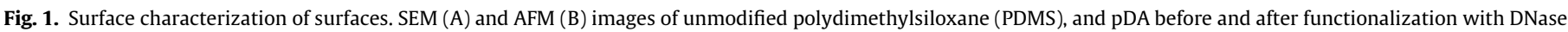

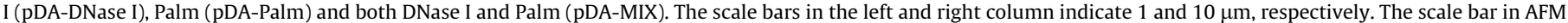
pictures indicates $1 \mu \mathrm{m}$.

agreement with reported studies [18,32]. Further functionalization with DNase I yielded surfaces with a heterogeneous roughness as the values measured within the same surface presented a large range, suggesting a non-uniform distribution of enzyme immobilization on the surface. On the other hand, and unlike in previous studies reporting other peptides immobilization using pDA as an intermediate layer [18,23,33], Palm immobilization decreased surface roughness which correlates with SEM results. When peptide was co-immobilized with enzyme (pDA-MIX), surface roughness increased which may be attributed to the presence of DNase I. To evaluate the surface wettability of the PDMS after surface modification, the static water contact angle of surfaces after each deposition step was measured (Fig. 2B). Bare PDMS is naturally hydrophobic, with a high contact angle of $109.9 \pm 3.0^{\circ}$. Its functionalization with pDA greatly improved the hydrophilicity of the polymeric surface, decreasing the contact angle to $56.6 \pm 4.8^{\circ}$, which is a well-established observation in other material surfaces as well $[23,34]$. Further immobilization with DNase I or Palm slightly increased the contact angle to $76 \pm 17^{\circ}$ and $77 \pm 15^{\circ}$, respectively, which may be attributed to the presence of hydrophobic amino acid residues in the enzyme [35] and Palm's hydrophobic lipophilic tail. Co-immobilization of enzyme and peptide (pDA-MIX) yielded surfaces more hydrophobic with a contact angle of $98 \pm 10^{\circ}$, which can be attributed to an addition effect provided by each compound. 

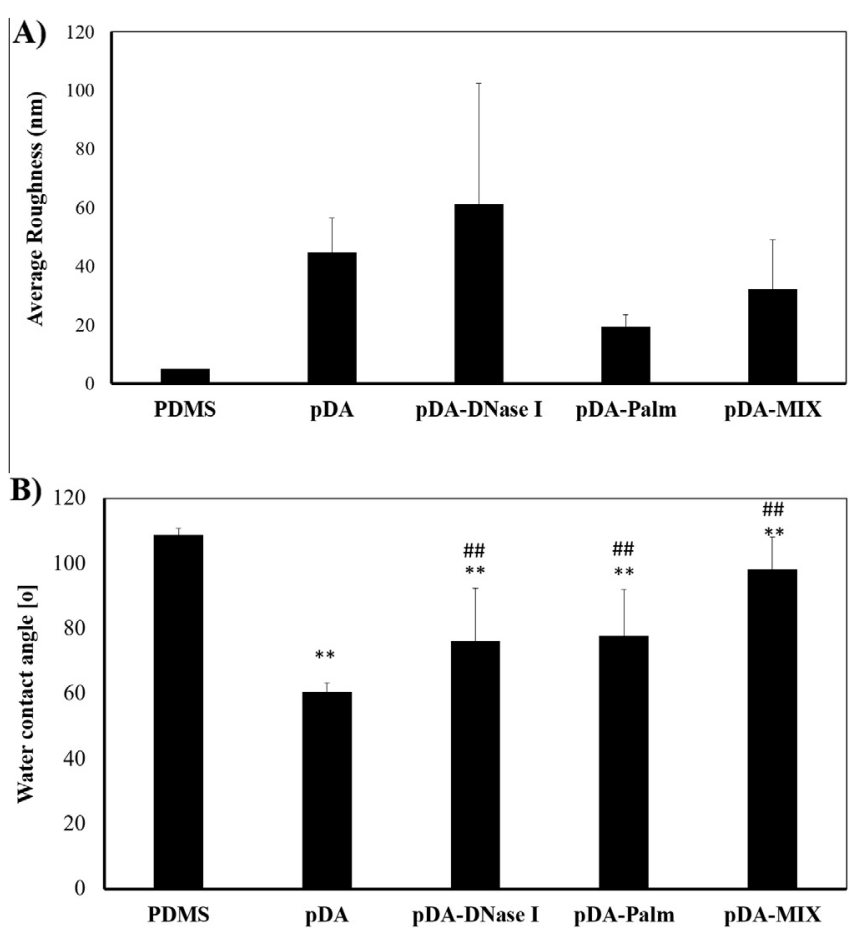

Fig. 2. Surface characterization of surfaces. Surface average roughness (A) and (B) water contact angles of unmodified polydimethylsiloxane (PDMS), and pDA before and after functionalization with DNase I (pDA-DNase I), Palm (pDA-Palm) and both DNase I and Palm (pDA-MIX). Significant differences were found for $(* *) \mathrm{p}<0.01$, compared to PDMS control and (\#\#)p < 0.01, compared to pDA control.

\subsection{Palm immobilization efficiency and stability}

To quantify the percentage of immobilized Palm, the buffer solutions containing the loading and unattached peptide were recovered after concluding the coating process. The fluorescamine assay revealed that $65.9 \% \pm 9.7 \%$ of loaded peptide was immobilized onto the pDA-coated PDMS surface and $56.7 \% \pm 2.1 \%$ of loaded Palm was still immobilized after being incubated in PBS for 5 days.

\subsection{Antibacterial performance of mono and bi-functional coatings}

Prior to co-immobilization of DNase I and Palm, their immobilization was performed alone. The antibacterial performance of these mono-functional coatings was investigated by performing an attachment assay in which bacteria were allowed to attach for $4 \mathrm{~h}$ and the remaining cells on the PDMS surfaces were imaged with fluorescence microscopy. In this assay, it was possible to measure the remaining cells on the modified surfaces and simultaneously discriminate between live and dead cells, or more, specifically, evaluate bacterial membrane's integrity (Fig. 3). For these analysis, a clinical isolate of $S$. aureus was chosen because of its clinical relevance. Bare PDMS surfaces allowed the adhesion of $S$. aureus cells, which have formed agglomerates, and most of them remained alive. Polydopamine-coated surfaces had no significant effect on bacterial attachment or cell viability but cells were more evenly distributed along these surfaces. Further functionalization with Palm, had no effect on bacterial attachment but was responsible for a greater fraction of dead cells. Enzymatic monofunctional coating, on the other hand, had no significant effect on cell viability as compared to bare PDMS surfaces but was able to
A)
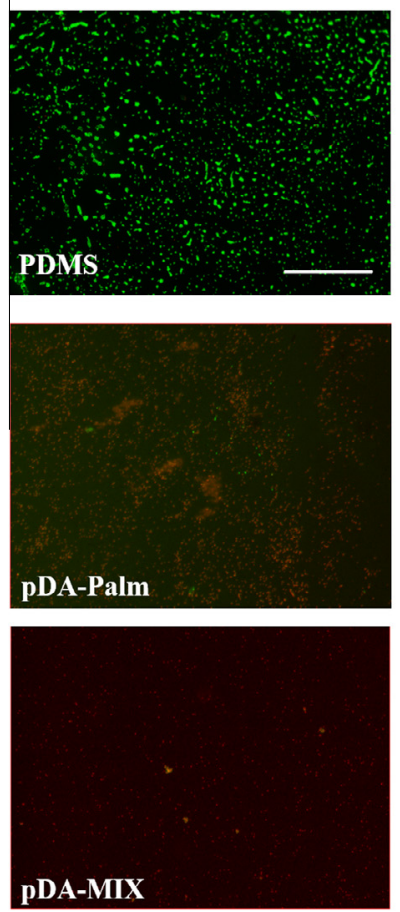

B)
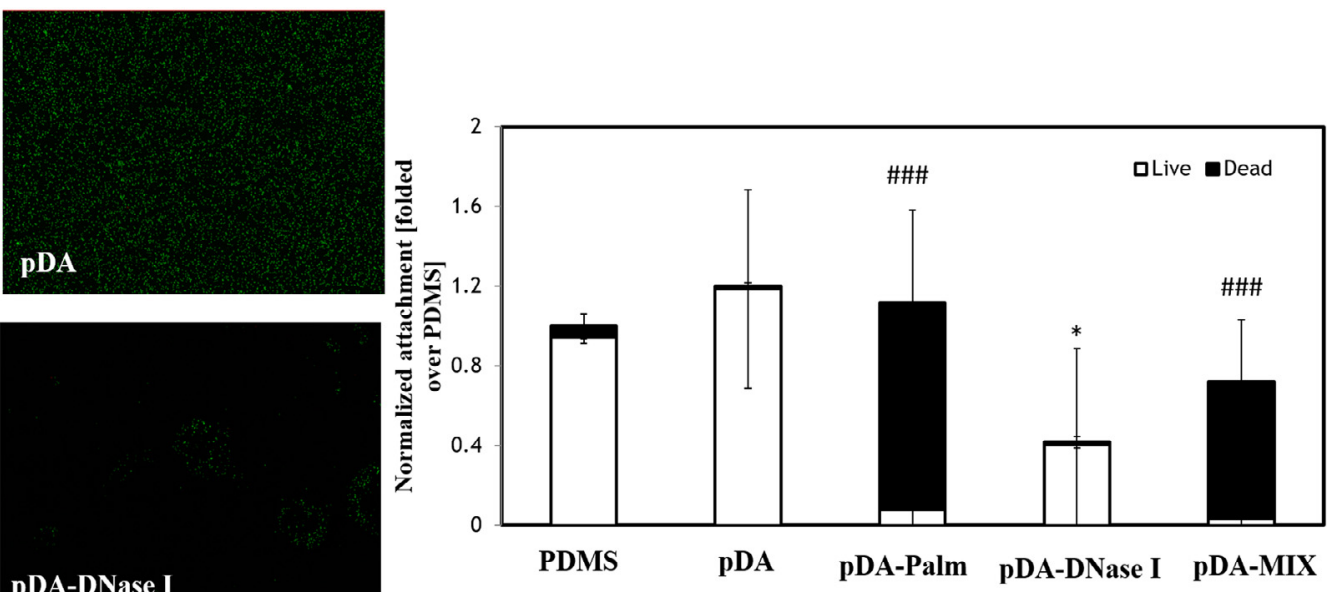

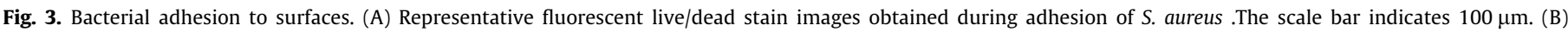

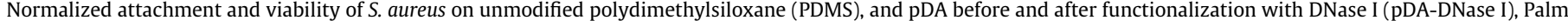

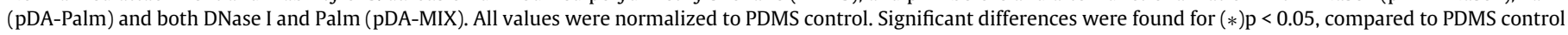
attachment and (\#\#\#)p $<0.001$, compared to PDMS fraction of dead cells. 
prevent bacterial attachment. Once confirmed the anti-adhesive and antimicrobial properties conferred by immobilization of DNase I and Palm, respectively, their co-immobilization was performed so that a bi-functional coating integrating both functionalities could be obtained (pDA-MIX). Results showed that with this combination, the fraction of dead cells greatly increased as compared to unmodified PDMS and bacterial attachment was slightly reduced as compared to PDMS functionalized with Palm alone, suggesting the additional effect conferred by the presence of DNase I.

\subsection{Adhesion of dual-species to mono and bi-functional coatings}

The performance of mono and bi-functional coatings proposed in the present study was also investigated against dual-species adhesion. For that, bacteria were allowed to attach for $4 \mathrm{~h}$ and the remaining cells on the PDMS surfaces were imaged with fluorescence microscopy after live/dead staining (Fig. 4). The fitness of dual-species adhesion (Fig. 4C) was compared to single-species adhesion (Fig. 4A and B) to mono-functional coatings. The clinical isolate of $S$. aureus and a reference strain of $P$. aeruginosa were used in this assay. As previously established, mono-functional coatings of DNase I was able to prevent $S$. aureus while Palm monofunctionalization generated surfaces able to kill most of bacteria (Fig. 4A). When it comes to $P$. aeruginosa adhesion to these mono-functional coatings, different antibacterial effects were observed. PDMS functionalized with pDA was able to prevent bacterial adhesion and also affected cell viability as compared to bare PDMS surfaces. PDMS surfaces functionalized with DNase I was able to prevent bacterial attachment while mono-functional coatings of Palm yielded similar results to pDA coating alone. Adhesion of $S$. aureus together with $P$. aeruginosa to bare PDMS surfaces yielded a higher fraction of dead cells as compared to their mono-species adhesion, suggesting an antagonistic interaction between bacterial strains. The presence of a pDA layer was responsible for reducing bacterial attachment. As $P$. aeruginosa was affected by the hydrophilic parameters of pDA, this results suggested that adhesion on bare PDMS was dominated by this strain. DNase I-based mono-functional coating was able to reduce bacterial attachment without significant effect on cell viability. These results are in accordance with the fitness observed for monospecies adhesion, as PDMS functionalized with DNase I was able to prevent the attachment of both strains, alone. Immobilization of Palm yielded interesting results as they were able to prevent bacterial attachment in a greater extent as compared to DNase I. These results also suggested that $P$. aeruginosa had some predominant effect on $S$. aureus adhesion. Co-immobilization of both peptide and enzyme at a proportion of 1:3 (pDA-MIX) yielded a bi-functional coating able to prevent bacterial attachment of both strains and increased the fraction of dead cells, as compared to bare PDMS surfaces. These results indicated that bi-functional coatings developed in this study retained their both antiadhesive and antimicrobial properties also against dual-species adhesion.

In order to confirm some of the hypothesis raised from aforementioned results, and also to directly visualize the location and distribution of bacterial strains within the dual-species community, adhesion of both strains, in the same conditions, was allowed to proceed on PDMS surfaces that were, afterwards, observed under a fluorescence microscope after applying a multiplex PNA FISH methodology counterstained with DAPI. The strength of each fluorescent signal allowed to distinguish $P$. aeruginosa (red rodshaped cells) and S. aureus (blue cocci) within the bacterial consortia (Fig. 4D). Results confirmed that $P$. aeruginosa was the dominant organism with $S$. aureus adhering afterwards on $P$. aeruginosa agglomerates. No significant differences could be observed on bacterial distribution on different PDMS modified surfaces.
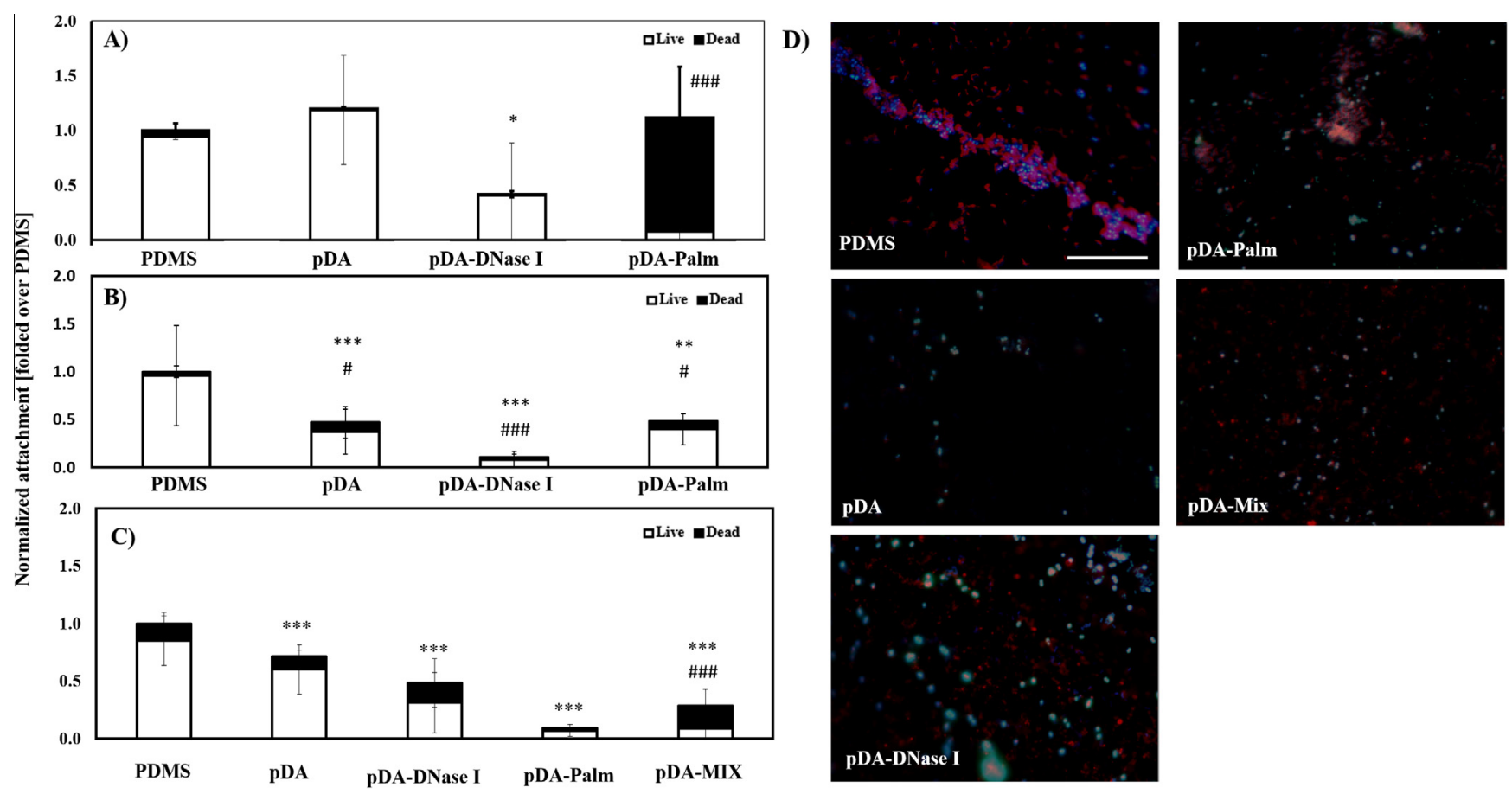

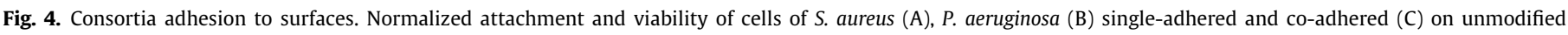

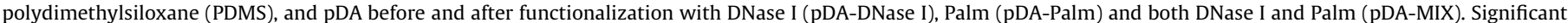

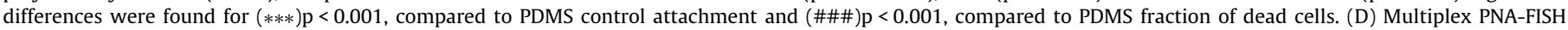

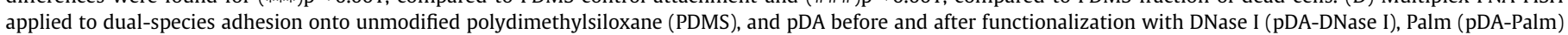
and both DNase I and Palm (pDA-MIX).The scale bar indicates $20 \mu \mathrm{m}$. 


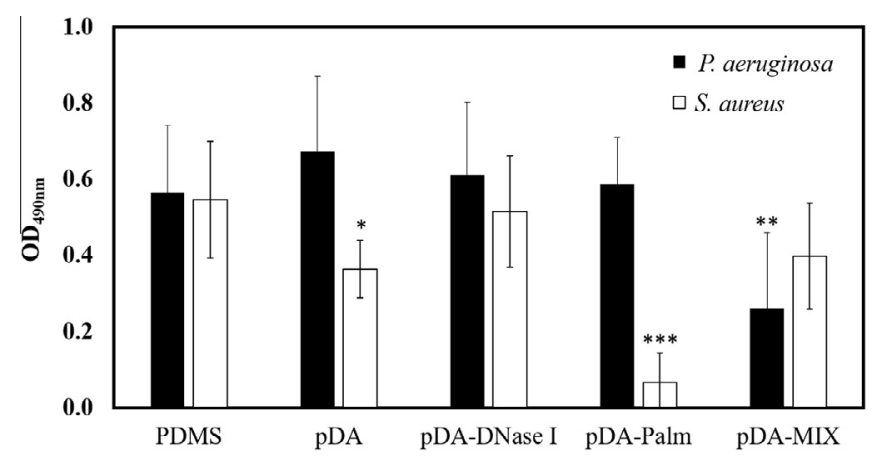

Fig. 5. Biofilm formation on surfaces. Metabolic activity of biofilm cells of $P$. aeruginosa (black) and $S$. aureus (white) adhered to unmodified polydimethylsiloxane (PDMS), and pDA before and after functionalization with DNase I (pDA-DNase I), Palm (pDA-Palm) and both DNase I and Palm (pDA-MIX). Significant differences were found for $(*) \mathrm{p}<0.05,(* *) \mathrm{p}<0.01$ and $(* * *) \mathrm{p}<0.001$, compared to PDMS control.

\subsection{Anti-biofilm properties of mono and bi-functional coatings}

To investigate the anti-biofilm properties of coatings, the clinical isolate of $S$. aureus and $P$. aeruginosa were allowed to grow as single bacteria, in TSB for $24 \mathrm{~h}$ and biofilm cells viability were evaluated using a XTT assay. In this assay, OD values are proportional to the number of metabolic active cells adhered on the surfaces. As shown in Fig. 5 unmodified PDMS exhibited the higher values of optical density, which confirms that PDMS surfaces are prone to both $S$. aureus or $P$. aeruginosa adhesion and subsequent biofilm formation. The presence of a pDA layer slightly decreased cell activity of $S$. aureus but had no effect on the metabolic activity of the Gram-negative strain. Mono-functional coatings of DNase I had no effect on biofilm formation of both strains but PDMS functionalized with Palm greatly decreased biofilm cells metabolic activity of $S$. aureus. Bi-functional coatings had no significant effect on biofilm cells metabolic activity of $S$. aureus but were able to compromise biofilm formation of $P$. aeruginosa.

\subsection{Evaluation of resistance development by bacterial cells adhered to modified PDMS surfaces}

In order to evaluate the potential of bacterial resistance development towards surfaces modified with Palm, an assay was performed in which cells in contact with unmodified PDMS and modified PDMS surfaces for a period of 10 days, were recovered and used to determine the MIC and MBC of Palm (Table 1). As a control, the antibiotic vancomycin was immobilized onto PDMS using dopamine chemistry, at the same concentration as the AMP and the same assay was performed.

Results showed that cells adhered to PDMS functionalized with Palm exhibited the same susceptibility pattern as cells adhered to PDMS before or after coating with pDA, suggesting no development

Table 1

MIC and MBC values of Palm and Vancomycin against adhered S. aureus recovered from unmodified PDMS and pDA before and after functionalization with Palm (pDAPalm) or vancomycin (pDA-Vancomycin).

\begin{tabular}{lll}
\hline Cells adhered to & MIC $(\mu \mathrm{g} / \mathrm{mL})$ & MBC $(\mu \mathrm{g} / \mathrm{mL})$ \\
\hline PDMS & 64 & $>64$ \\
pDA & 64 & $>64$ \\
pDA-Palm & 64 & $>64$ \\
PDMS & 1 & 1 \\
pDA & 1 & 2 \\
pDA-Vancomycin & 2 & 8 \\
\hline
\end{tabular}

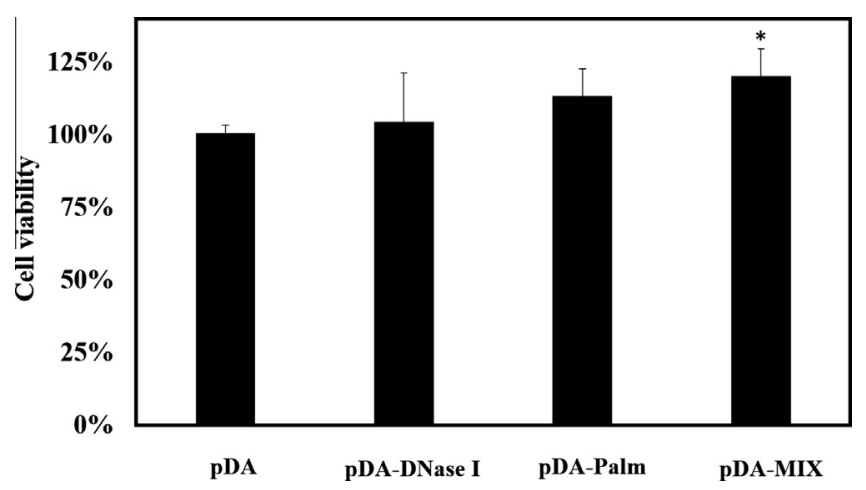

Fig. 6. Surfaces biocompatibility. Viability of $3 \mathrm{~T} 3$ fibroblast cells after $48 \mathrm{~h}$ of contact with pDA before and after functionalization with DNase I (pDA-DNase I), Palm (pDA-Palm) and both DNase I and Palm (pDA-MIX), measured with an MTS assay. Significant differences were found for $(*) \mathrm{p}<0.5$ compared to pDA control.

of resistance. On the other hand, cells adhered to surfaces functionalized with the antibiotic vancomycin were less susceptible to the same antibiotic with higher values of MIC and MBC as compared to PDMS unmodified or coated with pDA. The higher MBC of adhered cells when compared to planktonic culture was expected taking into consideration that adhered cells are less susceptible than their planktonic counterparts [36].

\subsection{Effect of PDMS modified surfaces on $3 T 3$ fibroblast metabolic activity}

To predict the effects of mono and bi-functional coatings developed in this study on mammalian cells, an assay of cytotoxicity was performed (Fig. 6). Results showed that further functionalization of pDA surfaces had no significant effect on 3T3 fibroblast metabolic activity. Bi-functional coatings slightly increased cell metabolic activity.

\section{Discussion}

The present study aimed to prepare a bi-functional coating incorporating the strengths of two strategies: to kill bacteria and simultaneously prevent their adhesion to surfaces, using a facile and non-toxic approach developed by Messersmith and co-workers [17]. The immobilization platform was performed on PDMS as this material has been widely used for the development of catheters [37]. A clinical isolate of $S$. aureus was used throughout this study as a model strain because biomaterial-associated infections are mainly caused by Gram-positive bacteria [38]. To confer surfaces with antimicrobial activity, the AMP Palm was chosen. Their potential as alternatives to conventional antibiotics, mainly due to their low propensity for inducing microbial resistance has been recognized in the past few years $[39,40]$. Palm belongs to a group of lipopeptides with powerful antifungal and antibacterial activities. These lipopeptides are obtained from positively charged peptides containing $\mathrm{D}$ - and L-amino acids (diastereomers) with palmitic acid at their $\mathrm{N}$ terminus [41]. As a lipopetide its mechanism of action consists of simple disruption of membrane electric potential [42]. Its immobilization did not compromise their antimicrobial activity as it may involve the displacement of positive cations from the bacterial membranes which induces disruption of the bacterial envelope and subsequent death [43]. Anti-adhesive component of bi-functional coatings was provided by the enzyme DNase I, targeting extracellular DNA. Because extracellular DNA facilitates the initial stage of bacterial adhesion to biomaterials and, virtually, all bacterial populations produce this structural component, it was hypothesized that it could be a 
general target [44]. Furthermore, the immobilization of DNase I onto biomaterial surfaces, using a pDA layer as an intermediate, has proved to be effective in preventing bacterial adhesion and biofilm formation up to $14 \mathrm{~h} \mathrm{[45].}$

Surface characterization studies confirmed the immobilization of Palm and DNase I onto pDA-coated PDMS surfaces and that most of the peptide did not detach from the surface for up to 5 days. Enzyme immobilization did not compromise its catalytic activity (data not shown). To assess the antibacterial performance of developed coatings, an adhesion assay was performed in which bacterial cells were allowed to adhere for $4 \mathrm{~h}$. This period of time was chosen because the first $6 \mathrm{~h}$ after surgery (the so-called "decisive period") are identified as being critical for preventing bacterial adhesion in order to assure the long-term success of the implant [46]. During this period of time, there is a race involving the attachment of bacteria and the incorporation of the material into the surrounding tissue [3]. Attachment assay confirmed that immobilization of Palm onto pDA-coated PDMS surfaces conferred them with potent antimicrobial properties given its ability to damage most of bacterial cells adhered to these coatings very fast. This performance is in accordance with lipopeptides ability to kill multi-resistant Gram-positive cocci, in solution, previously reported [47]. Enzymatic mono-functional coating was able to prevent bacterial attachment suggesting its suitability to confer the anti-adhesive properties intended for the bi-functional coating. Once confirmed the anti-adhesive and antimicrobial properties conferred by immobilization of DNase I and Palm, respectively, their co-immobilization was performed so that a bi-functional coating integrating both functionalities could be developed. Surface characterization of this bi-functional coating revealed a surface with intermediate roughness between the ones achieved with both compounds, alone. Co-immobilization of DNase I and Palm yielded more hydrophobic surfaces which can be attributed to an addition effect provided by each compound.

Most of the coating approaches developed to fight biomaterialassociated infections only investigate the antibacterial performance against bacterial strains as single species. In the present study, the performance of mono and bi-functional coatings against the adhesion of dual-species was also evaluated. Results confirmed that the bi-functional coating was able to prevent bacterial attachment of both strains and increase the fraction of damaged cells, as compare to unmodified PDMS. The coatings retained, therefore, both anti-adhesive and antimicrobial properties against dual-species adhesion.

Once established the potential of mono and bi-functional coatings to prevent bacterial attachment and kill bacteria adhered to the surfaces during the first stages of bacterial colonisation process, it is important to evaluate coatings efficacy to impair biofilm formation. For that, $S$. aureus or $P$. aeruginosa were allowed to grow in a nutrient-rich environment for $24 \mathrm{~h}$. Mono-functional coatings of DNase I had no effect on biofilm formation by both strains which may be attributed to the presence of proteases or macromolecules present in established biofilms which may have interfered with enzyme activity [5]. PDMS functionalized with Palm greatly decreased biofilm metabolic activity of $S$. aureus, suggesting, therefore, its ability to compromise biofilm establishment but had no effect against $P$. aeruginosa metabolic activity. These results correlate with the different susceptibility patterns obtained in planktonic cultures, as Palm was more effective against the Gram-positive strain. However, the mechanisms dictating these different performance should be further investigated.

The coatings developed in this study exhibited different performances, according to the type of media used, PBS or TSB, two extreme media in terms of composition. Therefore, the adhesion of $S$. aureus and $P$. aeruginosa, both as single and in a consortia, using synthetic urine [48], for a longer period of time, $24 \mathrm{~h}$, was also conducted. Preliminary results (Fig. S2) suggested that the bi-functional coatings were also effective using artificial urine and longer periods of time. These results highlight the promising application of these coatings on urinary catheters.

Bacteria exhibit an outstanding ability to adapt in response to their environment, and some bacterial strains demonstrated to be resistant to some AMP [49]. Because only a few works have addressed the development of bacterial resistance towards AMP after their immobilization [50], a study was performed on Palm-based coatings in order to evaluate the potential of bacterial resistance toward these surfaces. Results confirmed that Palm immobilization retained its low propensity to develop bacterial resistance, as opposite to the immobilization of an antibiotic.

\section{Conclusions}

In summary, a two-step polydopamine-based surface modification strategy was applied to successfully co-immobilize an antimicrobial peptide and an enzyme targeting an important component of biofilm matrix. This immobilization approach imparted PDMS surfaces with both anti-adhesive and antimicrobial properties against the adhesion of relevant bacteria as single and dualspecies, with excellent stability and biocompatible properties, holding, therefore, great potential in the development of catheters able to prevent CAI.

\section{Acknowledgements}

This study was supported by the Portuguese Foundation for Science and Technology (FCT) under the scope of the strategic funding of UID/BIO/04469/2013 unit and COMPETE 2020 (POCI01-0145-FEDER-006684). The authors also acknowledge the support by FCT and the European Community fund FEDER, through Program COMPETE, under the scope of the Projects AntiPep PTDC/SAU-SAP/113196/2009 (FCOMP-01-0124-FEDER-016012) and RECI/BBB-EBI/0179/2012 (FCOMP-01-0124-FEDER-027462) and the PhD Grant of Diana Alves (SFRH/BD/78063/2011). This study was also supported by the statutory fund from the Medical University of Gdansk (Project No. 02-0087/07/508).

\section{Appendix A. Supplementary data}

Supplementary data associated with this article can be found, in the online version, at http://dx.doi.org/10.1016/j.actbio.2016.08. 010.

\section{References}

[1] B.W. Trautner, R.O. Darouiche, Catheter-associated infections: pathogenesis affects prevention, Arch. Intern. Med. 164 (2004) 842-850.

[2] G. Subbiahdoss, R. Kuijer, D.W. Grijpma, H.C. van der Mei, H.J. Busscher, Microbial biofilm growth vs. tissue integration: "the race for the surface" experimentally studied, Acta Biomater. 5 (2009) 1399-1404.

[3] A.G. Gristina, Biomaterial-centered infection: microbial adhesion versus tissue integration, Science 237 (1987) 1588-1595.

[4] C.J. Nobile, A.P. Mitchell, Microbial biofilms: e pluribus unum, Curr. Biol. 17 (2007) R349-R353.

[5] H.C. Flemming, J. Wingender, The biofilm matrix, Nat. Rev. Microbiol. 8 (2010) 623-633.

[6] J.A. Niska, J.H. Shahbazian, R.I. Ramos, K.P. Francis, N.M. Bernthal, L.S. Miller, Vancomycin-rifampin combination therapy has enhanced efficacy against an experimental Staphylococcus aureus prosthetic joint infection, Antimicrob. Agents Chemother. 57 (2013) 5080-5086.

[7] L. Zhao, P.K. Chu, Y. Zhang, Z. Wu, Antibacterial coatings on titanium implants, Biomed. Mater. Res. B Appl. Biomater. 91 (2009) 470-480.

[8] D. Roe, B. Karandikar, N. Bonn-Savage, B. Gibbins, J.B. Roullet, Antimicrobial surface functionalization of plastic catheters by silver nanoparticles, J. Antimicrob. Chemother. 61 (2008) 869-876.

[9] P. Gao, X. Nie, M. Zou, Y. Shi, G. Cheng, Recent advances in materials for extended-release antibiotic delivery system, J. Antibiot. 64 (2011) 625-634. 
[10] K. Bazaka, M.V. Jacob, W. Chrzanowski, K. Ostrikov, Anti-bacterial surfaces: natural agents, mechanisms of action, and plasma surface modification, RSC Adv. 5 (2015) 48739-48759.

[11] D. Alves, M. Pereira, Mini-review: antimicrobial peptides and enzymes as promising candidates to functionalize biomaterial surfaces, Bioufouling 40 (2014) 483-499.

[12] E. Guani-Guerra, T. Santos-Mendoza, S.O. Lugo-Reyes, L.M. Teran, Antimicrobial peptides: general overview and clinical implications in human health and disease, Clin. Immunol. 135 (2010) 1-11.

[13] J.B. Kaplan, Biofilm dispersal: mechanisms, clinical implications, and potential therapeutic uses, J. Dent. Res. 89 (2010) 205-218.

[14] J.M. Goddard, J.H. Hotchkiss, Polymer surface modification for the attachment of bioactive compounds, Prog. Polym. Sci. 32 (2007) 698-725.

[15] J.C. Tiller, C.J. Liao, K. Lewis, A.M. Klibanov, Designing surfaces that kill bacteria on contact, Proc. Natl. Acad. Sci. U.S.A. 98 (2001) 5981-5985.

[16] J.F. Yala, P. Thebault, A. Hequet, V. Humblot, C.M. Pradier, J.M. Berjeaud, Elaboration of antibiofilm materials by chemical grafting of an antimicrobial peptide, Appl. Microbiol. Biotechnol. 89 (2011) 623-634.

[17] H. Lee, S.M. Dellatore, W.M. Miller, P.B. Messersmith, Mussel-inspired surface chemistry for multifunctional coatings, Science 318 (2007) 426-430.

[18] K. Lim, R.R. Chua, H. Bow, P.A. Tambyah, K. Hadinoto, S.S. Leong, Development of a catheter functionalized by a polydopamine peptide coating with antimicrobial and antibiofilm properties, Acta Biomater. 15 (2015) 127-138.

[19] G. Yeroslavsky, O. Girshevitz, J. Foster-Frey, D.M. Donovan, S. Rahimipour, Antibacterial and antibiofilm surfaces through polydopamine-assisted immobilization of lysostaphin as an antibacterial enzyme, Langmuir 31 (2015) 1064-1073.

[20] G.B. Fields, R.L. Noble, Solid phase peptide synthesis utilizing 9fluorenylmethoxycarbonyl amino acids, Int. J. Pept. Protein Res. 35 (1990) $161-214$.

[21] Methods for Dilution Antimicrobial Susceptibility Tests for Bacteria that Grow Aerobically, sixth ed., Clinical and Laboratory Standards Institute, Wayne, Pennsylvania, 2003.

[22] Y. Ren, J.G. Rivera, L. He, H. Kulkarni, D.K. Lee, P.B. Messersmith, Facile, high efficiency immobilization of lipase enzyme on magnetic iron oxide nanoparticles via a biomimetic coating, BMC Biotechnol. 11 (2011) 63.

[23] E. Ko, K. Yang, J. Shin, S.W. Cho, Polydopamine-assisted osteoinductive peptide immobilization of polymer scaffolds for enhanced bone regeneration by human adipose-derived stem cells, Biomacromolecules 14 (2013) 3202-3213.

[24] D.J. Stickler, J.C. Lear, N.S. Morris, S.M. Macleod, A. Downer, D.H. Cadd, W.J. Feast, Observations on the adherence of Proteus mirabilis onto polymer surfaces, J. Appl. Microbiol. 100 (2006) 1028-1033.

[25] R. Wang, K.G. Neoh, Z. Shi, E.T. Kang, P.A. Tambyah, E. Chiong, Inhibition of Escherichia coli and Proteus mirabilis adhesion and biofilm formation on medical grade silicone surface, Biotechnol. Bioeng. 109 (2012) 336-345.

[26] C. Almeida, N.F. Azevedo, S. Santos, C.W. Keevil, M.J. Vieira, Discriminating multi-species populations in biofilms with peptide nucleic acid fluorescence in situ hybridization (PNA FISH), PLoS One 6 (2011) e14786.

[27] S.P. Lopes, D.T. Carvalho, M.O. Pereira, N.F. Azevedo, Discriminating between typical and atypical cystic fibrosis-related bacterial species by multiplex PNA-FISH, Biotechnol. Bioeng. (2016). submitted for publication.

[28] R. Amann, B.M. Fuchs, Single-cell identification in microbial communities by improved fluorescence in situ hybridization techniques, Nat. Rev. Microbiol. 6 (2008) 339-348.

[29] M. Stevens, S. Olsen, Comparative-analysis of using MTT and XTT in colorimetric assays for quantitating bovine neutrophil bactericidal activity, J. Immunol. Methods 31 (1993) 261-271.

[30] J.N. Lee, X. Jiang, D. Ryan, G.M. Whitesides, Compatibility of mammalian cells on surfaces of poly(dimethylsiloxane), Langmuir 20 (2004) 11684-11691.
[31] A.P. Zhu, N. Fang, M.B. Chan-Park, V. Chan, Adhesion contact dynamics of 3T3 fibroblasts on poly (lactide-co-glycolide acid) surface modified by photochemical immobilization of biomacromolecules, Biomaterials 27 (2006) 2566-2576.

[32] D.R. Jun, S.K. Moon, S.W. Choi, Uniform polydimethylsiloxane beads coated with polydopamine and their potential biomedical applications, Colloids Surf. B 121 (2014) 395-399.

[33] K. Yang, J.S. Lee, J. Kim, Y.B. Lee, H. Shin, S.H. Um, J.B. Kim, K.I. Park, H. Lee, S.W. Cho, Polydopamine-mediated surface modification of scaffold materials for human neural stem cell engineering, Biomaterials 33 (2012) 6952-6964.

[34] T.S. Sileika, H.D. Kim, P. Maniak, P.B. Messersmith, Antibacterial performance of polydopamine-modified polymer surfaces containing passive and active components, ACS Appl. Mater. Interfaces 3 (2011) 4602-4610.

[35] W. Kabsch, H.G. Mannherz, D. Suck, E.F. Pai, K.C. Holmes, Atomic structure of the actin: DNase I complex, Nature 347 (1990) 37-44.

[36] S.D. Aaron, W. Ferris, K. Ramotar, K. Vandemheen, F. Chan, R. Saginur, Single and combination antibiotic susceptibilities of planktonic, adherent, and biofilm-grown Pseudomonas aeruginosa isolates cultured from sputa of adults with cystic fibrosis, J. Clin. Microbiol. 40 (2002) 4172-4179.

[37] R. Bayston, L.E. Fisher, K. Weber, An antimicrobial modified silicone peritoneal catheter with activity against both Gram-positive and Gram-negative bacteria, Biomaterials 30 (2009) 3167-3173.

[38] A. Vertes, V. Hitchins, K.S. Phillips, Analytical challenges of microbial biofilms on medical devices, Anal. Chem. 84 (2012) 3858-3866.

[39] M. Zasloff, Antimicrobial peptides of multicellular organisms, Nature 415 (2002) 389-395.

[40] M. Upton, P. Cotter, J. Tagg, Antimicrobial peptides as therapeutic agents, Int. J Microbiol. 2012 (2012) 1-2.

41] D. Avrahami, Y. Shai, A new group of antifungal and antibacterial lipopeptides derived from non-membrane active peptides conjugated to palmitic acid, J. Biol. Chem. 279 (2004) 12277-12285.

[42] P. Koszalka, E. Kamysz, M. Wejda, W. Kamysz, J. Bigda, Antitumor activity of antimicrobial peptides against U937 histiocytic cell line, Acta Biochim. Pol. 58 (2011) 111-117.

[43] R. Kugler, O. Bouloussa, F. Rondelez, Evidence of a charge-density threshold for optimum efficiency of biocidal cationic surfaces, Microbiology 151 (2005) $1341-1348$.

[44] T. Das, S. Sehar, M. Manefield, The roles of extracellular DNA in the structura integrity of extracellular polymeric substance and bacterial biofilm development, Environ. Microbiol. Rep. 5 (2013) 778-786.

[45] J.J.T.M. Swartjes, T. Das, S. Sharifi, G. Subbiahdoss, P.K. Sharma, B.P. Krom, H.J. Busscher, H.C. van der Mei, A functional DNase I coating to prevent adhesion of bacteria and the formation of biofilm, Adv. Funct. Mater 23 (2013) 2843-2849.

[46] K.A. Poelstra, N.A. Barekzi, A.M. Rediske, A.G. Felts, J.B. Slunt, D.W. Grainger, Prophylactic treatment of gram-positive and gram-negative abdominal implant infections using locally delivered polyclonal antibodies, J. Biomed. Mater. Res. 60 (2002) 206-215.

[47] W. Kamysz, C. Silvestri, O. Cirioni, A. Giacometti, A. Licci, A. Della Vittoria, M. Okroj, G. Scalise, In vitro activities of the lipopeptides palmitoyl (Pal)-Lys-Lys$\mathrm{NH}(2)$ and Pal-Lys-Lys alone and in combination with antimicrobial agents against multiresistant gram-positive cocci, Antimicrob. Agents Chemother. 51 (2007) 354-358.

[48] T. Brooks, C.W. Keevil, A simple artificial urine for the growth of urinary pathogens, Lett. Appl. Microbiol. 24 (1997) 203-206.

[49] J.L. Anaya-Lopez, J.E. Lopez-Meza, A. Ochoa-Zarzosa, Bacterial resistance to cationic antimicrobial peptides, Crit. Rev. Microbiol. 39 (2013) 180-195.

[50] D. Alves, M.O. Pereira, Bio-inspired coating strategies for the immobilization of polymyxins to generate contact-killing surfaces, Macromol. Biosci. (2016). 